\title{
$N$-acetylaspartate in the motor and sensory cortices following functional recovery after surgery for cervical spondylotic myelopathy
}

\author{
Sandy Goncalves, MSc, ${ }^{1,2}$ Todd K. Stevens, PhD, ${ }^{2}$ Patricia Doyle-Pettypiece, NP, MScN, ${ }^{3}$ \\ Robert Bartha, PhD, ${ }^{1,2}$ and Neil Duggal, MD, MSc, FRCS(C) $)^{1,3}$
}

\begin{abstract}
${ }^{1}$ Department of Medical Biophysics; ${ }^{2}$ Centre for Functional and Metabolic Mapping, Robarts Research Institute, Western University; and ${ }^{3}$ Department of Clinical Neurological Sciences, University Hospital, London Health Sciences Centre, London, ON, Canada
\end{abstract}

\begin{abstract}
OBJECTIVE Cervical spondylotic myelopathy (CSM) is the most common cause of reversible spinal cord dysfunction in people over the age of 55 years. Following surgery for symptomatic CSM, patients demonstrate motor improvement early in the postoperative course, whereas sensory improvement can lag behind. The authors of the present study hypothesized that changes in the concentration of $N$-acetylaspartate (NAA) in the motor and sensory cortices in the brain would emulate the time course of neurological recovery following decompression surgery for CSM. Their aim was to compare and contrast how metabolite levels in the motor and sensory cortices change after surgery to reverse downstream spinal cord compression.
\end{abstract}

METHODS Twenty-four patients with CSM and 8 control subjects were studied using proton MR spectroscopy $\left({ }^{1} \mathrm{H}\right.$ MRS) images acquired on a 3.0-T Siemens MRI unit. The ${ }^{1} \mathrm{H}-\mathrm{MRS}$ data (TE $135 \mathrm{msec}$, TR $2000 \mathrm{msec}$ ) were acquired to measure absolute levels of NAA from the motor and sensory cortices in the cerebral hemisphere contralateral to the side of greater deficit at baseline in each subject. Data were also acquired at 6 weeks and 6 months following surgery. Control subjects were also evaluated at 6 weeks and 6 months following baseline data acquisition. Neurological function was measured in each subject at all time points using the Neck Disability Index (NDI), modified Japanese Orthopaedic Association (mJOA) questionnaire, and the American Spinal Injury Association (ASIA) neurological classification.

RESULTS In the motor cortex of patients, NAA levels decreased significantly $(p<0.05)$ at 6 weeks and 6 months postsurgery compared with baseline levels. In the sensory cortex of patients, NAA levels decreased significantly $(p<0.05)$ only at 6 months after surgery compared with baseline and 6 -week levels. No significant changes in NAA were found in control subjects. Clinical scores demonstrated significant $(p<0.05)$ motor recovery by 6 weeks, whereas sensory improvements $(p<0.05)$ appeared at only 6 months.

CONCLUSIONS Findings suggest that metabolite changes in both the motor and sensory cortices mimic the time course of functional motor and sensory recovery in patients with CSM. The temporal course of neurological recovery may be influenced by metabolic changes in respective cortical regions.

http://thejns.org/doi/abs/10.3171/2016.2.SPINE15944

KEY WORDS cervical spondylotic myelopathy; motor; sensory; proton magnetic resonance spectroscopy

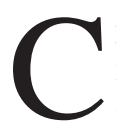
ERVICAL spondylotic myelopathy (CSM) is a common condition that is estimated to account for $2 \%$ of all hospital admissions and is the most frequent cause of spinal cord compression in patients older than 55 years. ${ }^{21}$ Symptoms begin to develop in a gradual but progressive stepwise manner and include sensory dysfunction, loss of dexterity, gait difficulties, and weakness in the upper and lower limbs. ${ }^{3,4,10,11,18,20}$ Following surgical decompression of the spinal cord, patients can typically experience early improvements in motor function and gait and unpredictable later improvements in sensory function..$^{1,16}$

Previous work by our group using proton MR spectroscopy $\left({ }^{1} \mathrm{H}-\mathrm{MRS}\right)$ has demonstrated a significant decrease in

ABBREVIATIONS ASIA = American Spinal Injury Association Impairment Classification; $\mathrm{Cr}=$ creatine; $\mathrm{CSM}=$ cervical spondylotic myelopathy; $\mathrm{Glu}=$ glutamate; ${ }^{1} \mathrm{H}-\mathrm{MRS}=$ proton MR spectroscopy; $\mathrm{mJOA}=$ modified Japanese Orthopaedic Association; NAA = N-acetylaspartate; NDI = Neck Disability Index.

SUBMITTED August 7, 2015. ACCEPTED February 26, 2016.

INCLUDE WHEN CITING Published online May 13, 2016; DOI: 10.3171/2016.2.SPINE15944. 
the $\mathrm{N}$-acetylaspartate/creatine (NAA/Cr) ratio in the motor cortex of CSM patients before surgery compared with that in healthy control subjects. ${ }^{14}$ Proton MRS is a noninvasive imaging technique that enables the measurement of high-concentration mobile metabolites such as NAA, $\mathrm{Cr}$, glutamate (Glu), choline, and myoinositol from specific volumes of brain tissue. ${ }^{19} \mathrm{~N}$-acetylaspartate is found in neurons as well as neuronal processes and is a marker of neuronal integrity and mitochondrial function. ${ }^{5,6}$ Cortical NAA is decreased in several pathological conditions such as Alzheimer's disease, bipolar disorder, stroke, and schizophrenia..$^{15}$

Our goal in the present study was to determine whether metabolite-level changes in the motor and sensory cortices mirror the pattern of motor and sensory functional changes observed in patients following surgery. Specifically, we hypothesized that early after surgery the concentration of NAA would change in the motor cortex while remaining unchanged in the sensory cortex and that by 6 months after surgery the NAA changes would be found in both the motor and sensory cortices, paralleling the time course of neurological recovery.

\section{Methods \\ Patient Population}

Twenty-four patients (20 males, 23 right handed, mean age \pm SEM $52 \pm 2$ years, range 34-67 years) with CSM and no other neurological impairments or previous surgical treatment in the brain or spinal cord were recruited to participate in this study. All participants provided written informed consent according to the Declaration of Helsinki, and the University of Western Ontario's Human Subjects Research Ethics Board approved this study. At the time of enrollment, all patients demonstrated progressive symptoms that had manifested no longer than 24 months. All patients presented with signal change at the site of compression on T2-weighted MRI. Patients underwent baseline MRI, surgical treatment, and 2 follow-up MRI examinations at 6 weeks and 6 months postsurgery that coincided with early and late clinical follow-up visits. Seven of the 24 patients were excluded from postoperative analyses because of attrition and data acquisition issues. Eleven of the remaining 17 patients were treated for single-level spinal cord compression, whereas 6 patients were treated for multilevel disease. We also recruited 8 control subjects of similar age (5 males, 8 right handed, mean age $48 \pm 3$ years) with no clinical history of CSM or previous surgery in the brain or spinal column. Control subjects also underwent 3 MRI examinations: at baseline and then after 6 weeks and 6 months.

All participants completed validated patient-derived clinical outcome measures including the modified Japanese Orthopaedic Association (mJOA) questionnaire and the Neck Disability Index (NDI). A physician or nurse practitioner assessed all participants using the physicianobjective American Spinal Injury Association Impairment Classification (ASIA) Scale.

\section{Magnetic Resonance Imaging}

All MRI was performed using a 3.0-T Siemens Magne- tom Tim Trio (15 patients) or a 3.0-T Siemens Magnetom Prisma Fit (2 patients) unit with a 32-channel head coil. Each examination (total scan time 48 minutes for patients and 62 minutes for control subjects) included the acquisition of sagittal 3D T1-weighted inversion-prepared (TI $900 \mathrm{msec}$ ) rapid gradient echo anatomical images (192 slices, $1 \mathrm{~mm}$ isotropic resolution, TR $2300 \mathrm{msec}$, TE 3.42 $\mathrm{msec}$ ) over the whole brain. These images produced high gray matter/white matter/cerebral spinal fluid contrast and were used for the measurement of tissue partial volume within the spectroscopy voxel.

A 20-mm isotropic voxel was positioned in the precentral gyrus over the area of the motor cortex that controls hand function, as defined by Yousry et al. ${ }^{22}$ using functional activation studies. Anatomical landmarks were also used to place the voxel. In the sagittal view the hand region of the motor cortex features the characteristic hook or "knob" appearance of the precentral gyrus, while in the axial view the cortex appears in the shape of an omega (Fig. 1). A second spectroscopy voxel was placed in the sensory cortex, immediately posterior to the voxel placed in the motor cortex. Three-dimensional images of the position of both voxels were saved for each participant to aid in placing the voxel over the same area during follow-up visits. Voxels were placed in the motor and sensory cortices in the hemisphere contralateral to the side of increased clinical deficit (on the right in 3 patients, on the left in 14 patients), while spectra for control subjects were acquired in the motor and sensory cortices on both sides of the brain. Data were not acquired on both sides of the brain in patients because of discomfort in the arms and neck due to CSM. Water-suppressed spectroscopic data were localized using point-resolved spectroscopy (PRESS; TR $2000 \mathrm{msec}$, TE $135 \mathrm{msec}$, averages 192, voxel size $8 \mathrm{~cm}^{3}$ ), and water-unsuppressed spectroscopic data were acquired from the same voxel.

Prior to spectral analysis, residual water signal was removed by subtraction using a Lanczos algorithm for singular value decomposition. ${ }^{13}$ Resultant spectra were fitted in the time domain using a Levenberg-Marquardt minimization routine incorporating a template of prior knowledge of metabolite lineshapes. In-house analysis software (fitMAN), created in our laboratory in the IDL (version 5.4, Research Systems Inc.) programming language, was used to process and visualize all spectra. ${ }^{2}$

Tissue partial volume analysis was performed using the T1-weighted anatomical images for each individual to determine the fraction of tissue and CSF within the voxel. Metabolite levels were normalized to the total tissue water signal and adjusted to account for water- and metabolitespecific $\mathrm{T} 1$ and $\mathrm{T} 2$ relaxation. The relaxation time constants were obtained from the literature from measurements made at 3.0T. According to previous studies, NAA is the primary metabolite involved in CSM. For the sake of completeness, the other major metabolites contributing to the spectrum were measured and reported if the group coefficient of variation was repeatedly $<50 \%$ : choline, $\mathrm{Cr}$, myoinositol, Glu.

\section{Statistical Analysis}

Absolute concentrations of metabolites were compared 
between groups using a 2-tailed Student t-test with an alpha error of 0.05 . For comparisons at different time points (baseline, 6 weeks, and 6 months) in patients, a 2-tailed paired Student t-test with an alpha error of 0.5 was also used. Correlations between clinical scores and absolute metabolite concentrations were determined using the Pearson correlation coefficient (r). Statistical analysis does not account for multiple comparisons due to the exploratory nature of this study as well as the planned comparisons made during conception of this study.

\section{Results}

\section{Clinical Outcome Measures}

Table 1 summarizes patient and control demographic information as well as the clinical outcome measurements made using the NDI, mJOA, and ASIA scales. Patients participating in the study demonstrated a significantly decreased NDI score $(p<0.05)$ from baseline to 6 months after surgery, indicating an improvement in neck pain and its effect on daily activities. A significant increase was observed in global mJOA scores from baseline to 6 months $(\mathrm{p}<0.001)$ and from 6 weeks to 6 months $(\mathrm{p}<0.05)$, indicating improvement in both motor and sensory function. A trend toward an increase $(p=0.07)$ was also found in the mJOA score when comparing baseline to 6-week global scores. A breakdown of the motor and sensory scores of the mJOA questionnaire can be found in Table 1. Patients showed a significant improvement in ASIA scores, the only physician-derived clinical measure. The ASIA classification can be subdivided into 2 categories, a motor component and a sensory component. In the motor subsection, there was significant improvement between baseline and 6 weeks $(\mathrm{p}<0.05)$ and between baseline and 6 months $(\mathrm{p}<$ $0.05)$. There was no statistical change in motor scores between 6 weeks and 6 months. In the sensory section of the ASIA classification, specifically with regard to light touch, there was significant improvement between baseline and 6 months ( $\mathrm{p}<0.01)$ and between 6 weeks and 6 months ( $\mathrm{p}$ $<0.05)$. There was no significant change between baseline and 6 weeks.

\section{Metabolic Changes in the Motor and Sensory Cortices}

The absolute concentration of NAA remained stable in control subjects across all 3 time points (baseline, 6 weeks, and 6 months) in both the motor and sensory cortices. Table 2 describes the absolute metabolite levels in patients with CSM at each time point (baseline and 6 weeks and 6 months postsurgical treatment) in both cortices. The concentration of NAA changed over time in both the motor and sensory cortices (Fig. 2). Specifically, NAA showed a significant decrease in the motor cortex from baseline to 6 weeks postoperatively $(\mathrm{p}<0.05)$ and from baseline to 6 months postoperatively $(\mathrm{p}=0.05)$. There was no change in NAA between 6 weeks and 6 months postoperatively. Interestingly, a different profile was observed in the sensory cortex, with NAA showing a significant decrease between 6 weeks and 6 months postoperatively $(\mathrm{p}<0.05)$ and from baseline to 6 months $(\mathrm{p}<0.05)$. There was no change in NAA in the sensory cortex in the first 6 weeks following surgery.
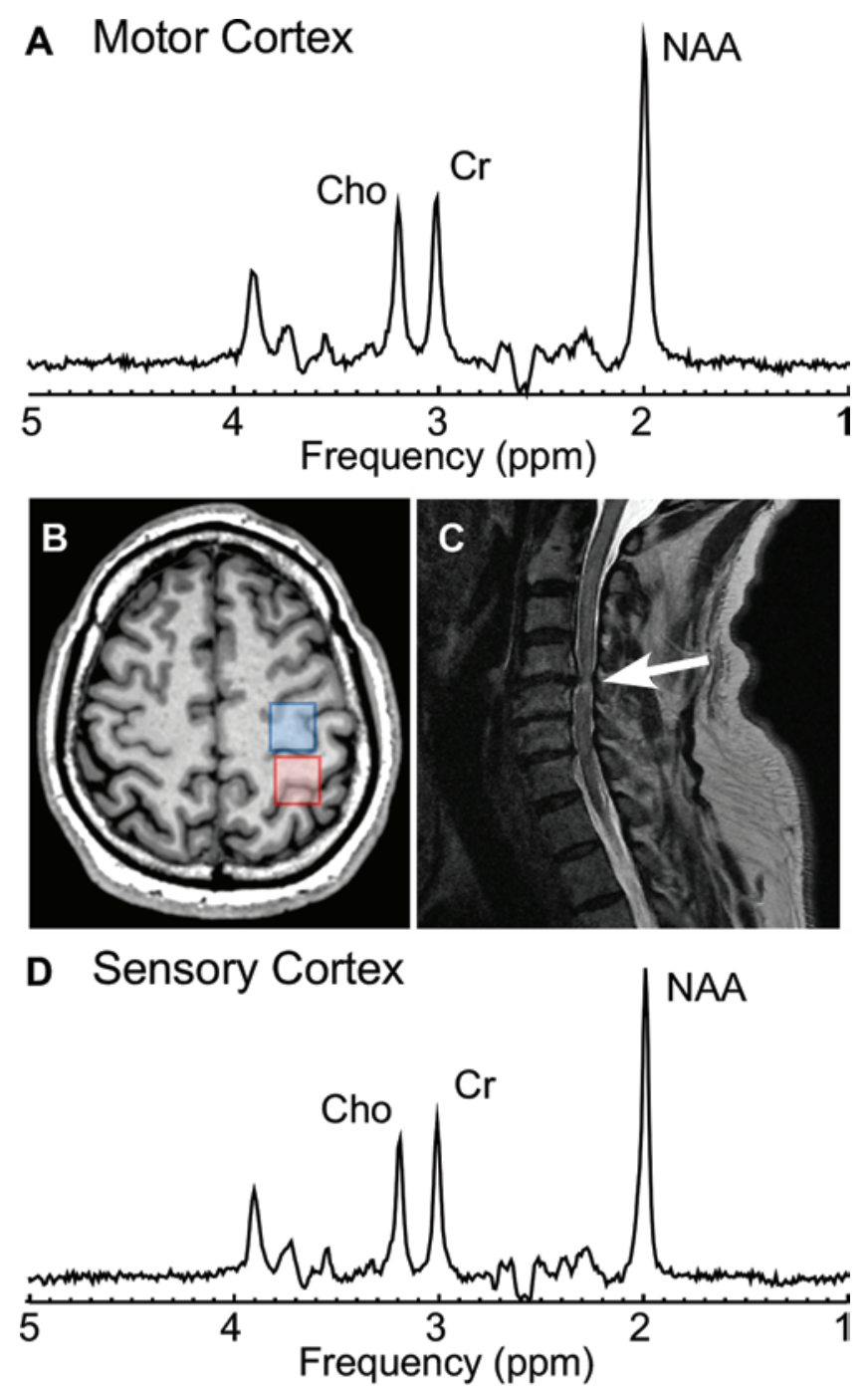

FIG. 1. A: A typical ${ }^{1} \mathrm{H}-\mathrm{MR}$ spectrum from the hand area of the motor cortex obtained from a patient with CSM. B: An axial T1-weighed MR image showing typical voxel placements incorporating the motor and sensory cortices. The $20 \times 20 \times 20-\mathrm{mm}$ spectroscopy voxel is placed on the "precentral knob" of the motor cortex (blue box). An identical voxel is placed over the sensory cortex (red box). C: A representative clinical sagittal cervical spine MR image demonstrating signal change in the spine (white arrow indicates the site of compression) with multilevel disease. D: A typical ${ }^{1} \mathrm{H}-\mathrm{MR}$ spectrum from the sensory cortex of a patient with CSM. Cho = choline. Figure is available in color online only.

No significant differences were detected $(\mathrm{p}>0.05)$ when comparing the absolute concentrations of the other individual metabolites (Table 2) in either the motor or sensory cortices across all 3 time points. To exclude any changes in metabolites that could be attributed to differences in single-level versus multilevel disease, a post hoc comparison was made between the 2 groups. No significant changes were found in metabolites when comparing patients with single-level and multilevel disease in both the motor and sensory cortices.

Comparing the changes in NAA levels with the changes in clinical scores, a significant correlation was found between the change in the motor portion of the mJOA af- 
TABLE 1. Demographic and clinical data for patients and controls

\begin{tabular}{|c|c|c|c|c|c|}
\hline Parameter & \multicolumn{2}{|c|}{ Patients } & \multicolumn{2}{|c|}{ Controls } & $p$ Value \\
\hline No. & \multicolumn{2}{|c|}{17} & \multicolumn{2}{|c|}{8} & NA \\
\hline Age & \multicolumn{2}{|c|}{$52 \pm 2$} & \multicolumn{2}{|c|}{$48 \pm 3$} & 0.230 \\
\hline Sex (male/female) & \multicolumn{2}{|c|}{$16 / 1$} & \multicolumn{2}{|c|}{$5 / 3$} & 0.136 \\
\hline Handedness (rt/lt) & \multicolumn{2}{|c|}{$16 / 1$} & \multicolumn{2}{|c|}{$8 / 0$} & 0.332 \\
\hline \multicolumn{6}{|l|}{ NDI scores* } \\
\hline Baseline & \multicolumn{2}{|c|}{$19.2 \pm 2.0$} & \multicolumn{2}{|c|}{$2.3 \pm 0.8$} & $<0.0001$ \\
\hline 6 wks & \multicolumn{2}{|c|}{$15.9 \pm 1.8$} & \multicolumn{2}{|c|}{$2.5 \pm 0.9$} & $<0.0001$ \\
\hline $6 \mathrm{mos}$ & \multicolumn{2}{|c|}{$12.8 \pm 2.1$} & \multicolumn{2}{|c|}{$2.9 \pm 1.0$} & 0.0002 \\
\hline \multicolumn{6}{|l|}{ mJOA scores } \\
\hline \multicolumn{6}{|l|}{ Baseline } \\
\hline Motor questions & \multicolumn{2}{|c|}{$8.3 \pm 2.5$} & \multicolumn{2}{|c|}{$12 \pm 0$} & $<0.0001$ \\
\hline Sensory questions & \multicolumn{2}{|c|}{$4.2 \pm 0.8$} & \multicolumn{2}{|c|}{$6 \pm 0$} & $<0.0001$ \\
\hline Global score & \multicolumn{2}{|c|}{$12.4 \pm 2.9$} & \multicolumn{2}{|c|}{$18 \pm 0$} & $<0.0001$ \\
\hline \multicolumn{6}{|l|}{6 wks } \\
\hline Motor questions & \multicolumn{2}{|c|}{$9.4 \pm 2.1$} & & & $<0.0001$ \\
\hline Sensory questions & & 1.0 & & & $<0.0001$ \\
\hline Global score & & 2.5 & & & $<0.0001$ \\
\hline $6 \mathrm{mos}$ & & & & & \\
\hline Motor questions & & 1.8 & & & $<0.0001$ \\
\hline Sensory questions & & 0.6 & & & $<0.0001$ \\
\hline Global score & & 2.2 & & & $<0.0001$ \\
\hline ASIA scores & Rt & $\mathrm{Lt}$ & Rt & Lt & \\
\hline Baseline & & & & & \\
\hline Upper motor & $23.5 \pm 0.4$ & $24.2 \pm 0.4$ & $25 \pm 0$ & $25 \pm 0$ & 0.0016 \\
\hline Lower motor & $23.8 \pm 0.4$ & $24.3 \pm 0.3$ & $25 \pm 0$ & $25 \pm 0$ & 0.0012 \\
\hline Light touch & $24.5 \pm 1.3$ & $25.8 \pm 1.2$ & $30 \pm 0$ & $30 \pm 0$ & 0.0007 \\
\hline Pin prick & $24.5 \pm 1.2$ & $25.2 \pm 1.3$ & $30 \pm 0$ & $30 \pm 0$ & 0.0005 \\
\hline 6 wks & & & & & \\
\hline Upper motor & $24.7 \pm 0.2$ & $24.8 \pm 0.1$ & $25 \pm 0$ & $25 \pm 0$ & 0.0460 \\
\hline Lower motor & $24.6 \pm 0.3$ & $24.8 \pm 0.1$ & $25 \pm 0$ & $25 \pm 0$ & 0.2261 \\
\hline Light touch & $26.8 \pm 1.0$ & $28.2 \pm 0.7$ & $30 \pm 0$ & $30 \pm 0$ & 0.0029 \\
\hline Pin prick & $27.0 \pm 1.0$ & $28.5 \pm 0.7$ & $30 \pm 0$ & $30 \pm 0$ & 0.0041 \\
\hline $6 \mathrm{mos}$ & & & & & \\
\hline Upper motor & $24.7 \pm 0.2$ & $24.9 \pm 0.1$ & $25 \pm 0$ & $25 \pm 0$ & 0.0488 \\
\hline Lower motor & $24.8 \pm 0.1$ & $24.8 \pm 0.2$ & $25 \pm 0$ & $25 \pm 0$ & 0.1300 \\
\hline Light touch & $29.3 \pm 0.3$ & $28.8 \pm 0.5$ & $30 \pm 0$ & $30 \pm 0$ & 0.0100 \\
\hline Pin prick & $29.0 \pm 0.7$ & $28.8 \pm 0.5$ & $30 \pm 0$ & $30 \pm 0$ & 0.0304 \\
\hline
\end{tabular}

$\mathrm{NA}=$ not applicable.

* Raw scores.

ter 6 months and the corresponding change in the absolute concentration of NAA in the motor cortex $(r=0.44, p=$ 0.04 ; Fig. 3). A similar temporal pattern was observed in significant changes between time points in the motor portion of the ASIA questionnaire and the concentration of NAA in the motor cortex (Fig. 4). Likewise, significant changes between time points in the concentration of NAA in the sensory cortex had a similar temporal pattern to changes in the sensory portion of the ASIA questionnaire when testing light touch (Fig. 5).

\section{Discussion}

This study is the first to characterize the distinct metabolic profile in the motor and sensory cortices in patients with CSM at baseline, 6 weeks postsurgery, and 6 months postsurgery. Clinical outcome measures were collected and neurological exams were performed at all 3 time points, providing a clinical context for the metabolite level measurements. In previous CSM studies, NAA metabolite levels were normalized to levels of $\mathrm{Cr}$, but differences 
TABLE 2. Absolute metabolite concentrations for patient group*

\begin{tabular}{|c|c|c|c|c|c|c|}
\hline \multirow[b]{2}{*}{ Metabolite } & \multicolumn{3}{|c|}{ Motor Cortex } & \multicolumn{3}{|c|}{ Sensory Cortex } \\
\hline & Baseline & $6 \mathrm{Wks}$ & 6 Mos & Baseline & $6 \mathrm{Wks}$ & 6 Mos \\
\hline NAA & $15.7 \pm 0.4$ & $14.9 \pm 0.6 \dagger$ & $14.5 \pm 0.5 \dagger$ & $15.8 \pm 0.7$ & $15.8 \pm 0.5$ & $14.6 \pm 0.5 \dagger \ddagger$ \\
\hline Glu & $4.4 \pm 0.3$ & $3.8 \pm 0.3$ & $3.8 \pm 0.2$ & $4.8 \pm 0.4$ & $4.5 \pm 0.4$ & $4.2 \pm 0.3$ \\
\hline $\mathrm{Cr}$ & $8.6 \pm 0.4$ & $8.3 \pm 0.4$ & $7.9 \pm 0.3$ & $8.6 \pm 0.3$ & $8.9 \pm 0.5$ & $8.3 \pm 0.3$ \\
\hline Cho & $2.5 \pm 0.1$ & $2.5 \pm 0.1$ & $2.4 \pm 0.1$ & $2.6 \pm 0.1$ & $2.8 \pm 0.1$ & $2.5 \pm 0.1$ \\
\hline $\mathrm{ml}$ & $2.6 \pm 0.3$ & $2.4 \pm 0.1$ & $2.3 \pm 0.1$ & $2.4 \pm 0.4$ & $2.4 \pm 0.3$ & $2.2 \pm 0.2$ \\
\hline
\end{tabular}

found in the NAA/Cr ratio over time do not necessarily reflect a change in the concentration of NAA. A temporal change may be caused by an alteration in the concentration of NAA, Cr, or both. Thus, to avoid uncertainty, we quantified the absolute levels of each metabolite.

After surgery, absolute NAA levels in the motor cortex decreased in the first 6 weeks following surgery and remained depressed at 6 months postsurgery. In contrast, in the sensory cortex, there was a delayed decrease in absolute NAA concentrations occurring between 6 weeks and 6 months after surgery. This change paralleled the recovery in motor function observed in the first 6 weeks and the sensory improvements that occurred later in the postoperative period between 6 weeks and 6 months.

The mJOA, ASIA, and NDI clinical outcome measures all demonstrated a significant improvement across time points. Patients consistently improved in motor as well as sensory function on both patient-subjective and physicianobjective measures. We found a significant correlation between the decrease in the absolute concentration of NAA in the motor cortex and the improvement in clinical function as measured by the motor function component of the mJOA neurological classification between baseline and 6 months.

In a previous study by our group,${ }^{14}$ we found decreased

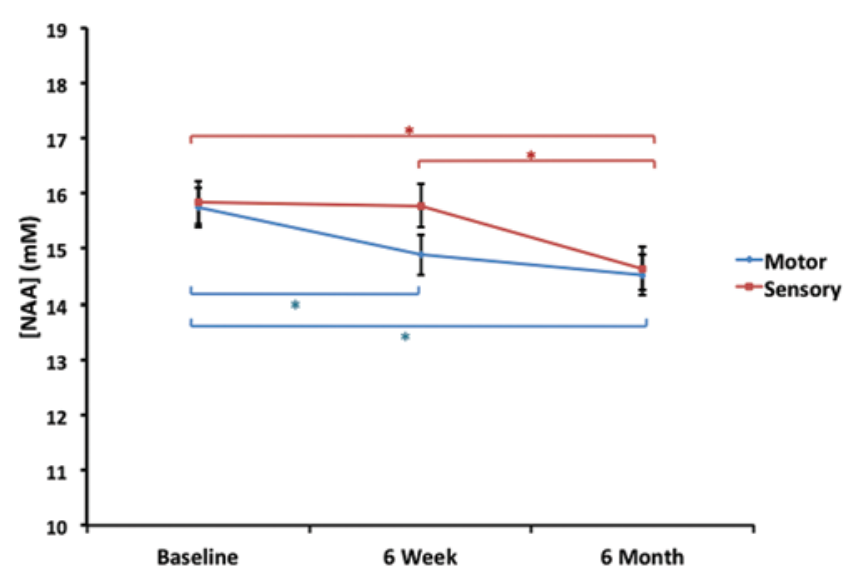

FIG. 2. The change in average absolute concentrations of NAA in patients with CSM following surgery. Error bars represent the standard error of the mean, and asterisks represent significant differences between time points. Figure is available in color online only. preoperative NAA/Cr levels in the motor cortex in CSM patients. The current study provides additional insight into the impact of surgical intervention and the time course of changes that occur in NAA levels in the motor and sensory cortices. We are the first to report that absolute NAA concentrations in the cortex decrease following surgery. It is unclear whether these metabolic changes are permanent (suggesting neuronal or axonal loss) or whether reduced NAA indicates mitochondrial dysfunction that is potentially reversible. ${ }^{7}$ Of note, similar findings using ${ }^{1} \mathrm{H}-\mathrm{MRS}$ of the cervical spinal cord have been reported by Salamon et al. ${ }^{17}$ and Ellingson et al., ${ }^{9}$ suggesting decreases in NAA account for axonal loss and decreased metabolic function.

When comparing decreases in the absolute concentration of NAA between the motor and sensory cortices, a temporal difference was observed. The decline in NAA levels in the motor cortex was most pronounced between baseline (presurgery) and 6 weeks postsurgery. A significant improvement was observed between the motor component of the ASIA at 6 weeks and 6 months compared with baseline. There was no significant improvement in the motor component of the ASIA scores between 6 weeks and 6 months. This pattern mirrored the decrease in the

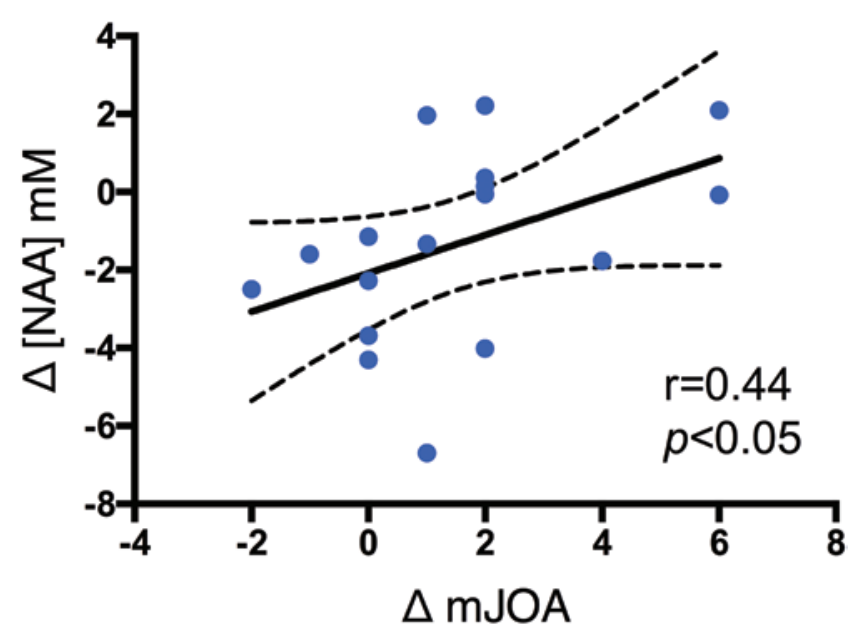

FIG. 3. Correlation between the change in scores on the motor portion of the mJOA questionnaire and the change in the absolute concentration of NAA in the motor cortex 6 months after surgery. A linear regression line (solid line) is shown superimposed on the data. Figure is available in color online only. 


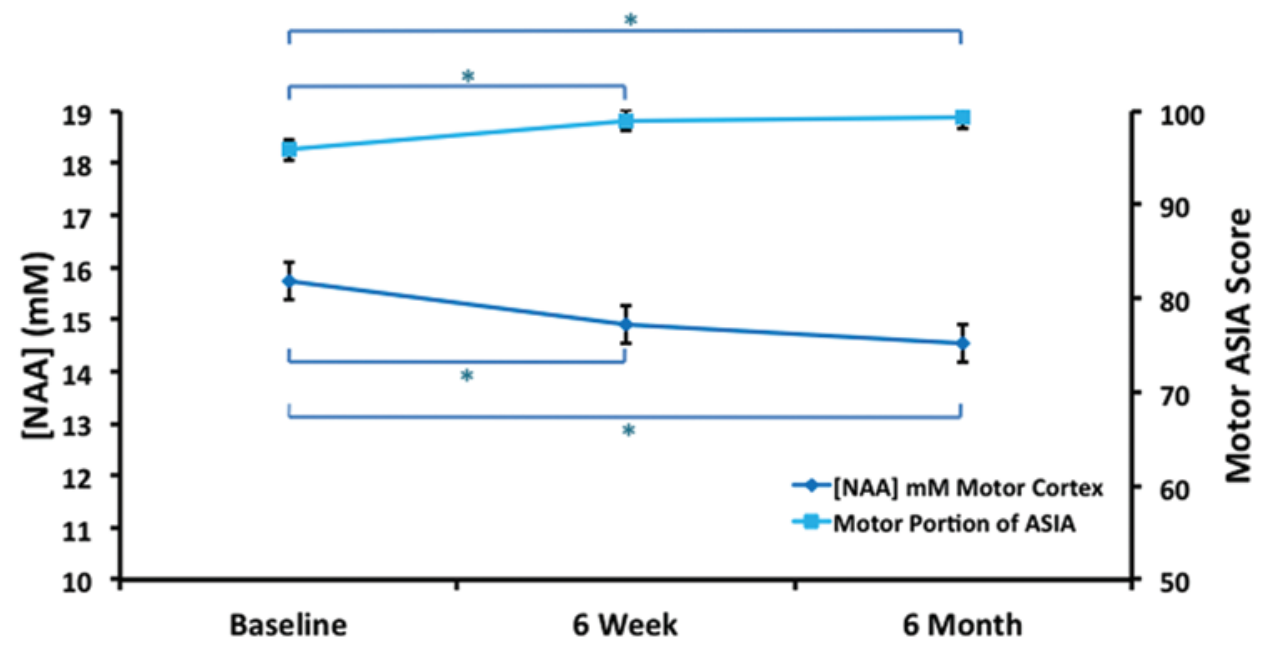

FIG. 4. The change in average absolute concentrations of NAA and average motor ASIA scores in patients with CSM following surgery. Error bars represent the standard error of the mean, and asterisks represent significant differences between time points. Figure is available in color online only.

absolute concentration of NAA in the motor cortex (Fig. 4). The significant correlation between the decrease in NAA and the improvement in motor function 6 months following surgery (Fig. 3) indicates that individuals with the greatest improvement also had the smallest decline in NAA levels.

In the sensory cortex, the significant decline in NAA levels occurred between 6 weeks and 6 months postsurgery. Compared with presurgery, a decline in NAA levels was also observed in the sensory cortex, but only 6 months after surgery. In contrast to the motor subsection of the ASIA, a different pattern was observed in the sensory component of the ASIA. The sensory component of the ASIA was significantly improved at 6 months as compared with both baseline and 6 weeks postsurgery. There was no significant improvement in sensory-specific ASIA scores between baseline and 6 weeks. This pattern also mirrored the decrease observed in the absolute concentration of NAA in the sensory cortex (Fig. 5). Therefore, the
NAA decline in the motor cortex preceded that observed in the sensory cortex.

The clinical dichotomy of motor and sensory symptom recovery demonstrated in the current study has been shown in 2 other studies. Moussellard et al. ${ }^{16}$ reported a consistent recovery in motor symptoms at the first followup (1 month postsurgery) and a subsequent plateau later in recovery. Al Tamimi et al. ${ }^{1}$ described unpredictable sensory symptom recovery at $3,12,24$, and 60 months postsurgery. While the limited sample size of the current study prevents data correlation between ${ }^{1} \mathrm{H}-\mathrm{MRS}$ and specific motor and sensory symptoms to demonstrate a direct relationship, our results encourage further investigation to uncover the temporal-metabolic process occurring in the motor and sensory neurons of the cortex that would otherwise be indistinguishable by current practice.

Previous studies have shown that damaged neurons in the sensorimotor cortex may undergo adaptive changes

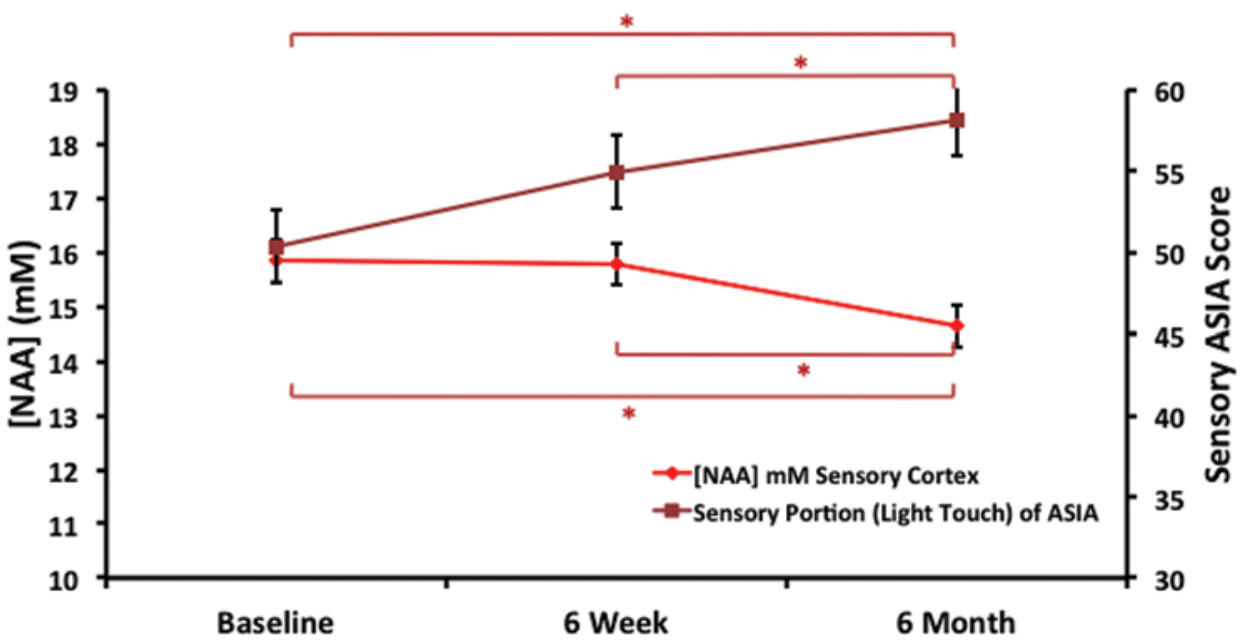

FIG. 5. The change in average absolute concentrations of NAA and average sensory (light touch) ASIA scores in patients with CSM following surgery. Error bars represent the standard error of the mean, and asterisks represent significant differences between time points. Figure is available in color online only. 
following surgical decompression in patients with CSM followed by neurological recovery. ${ }^{8,12}$ The exact trigger of cortical reorganization in adjacent neurons remains unclear. One possible explanation is that neurons experience synaptic modification in which preexisting connections develop new circuitry to restore lost function. ${ }^{12}$ Results from the current study demonstrate that despite successful surgical decompression and clinical recovery, NAA levels decline, suggesting not only neuronal impairment, but also, more specifically, dysfunction in neuronal mitochondria. ${ }^{5,6}$ Although this study reveals that metabolite level changes can be detected in both the motor and sensory cortices, future studies are needed to determine whether the temporal decreases in absolute NAA levels are the trigger for the recruitment of surrounding cortex and/or if decreasing NAA levels are a consequence of decreased metabolic demand due to cortical reorganization in which adjacent neurons are now incorporated into primary circuitry. This notion implies that observed temporal NAA level decreases are a product of an unknown mechanism triggering early motor function recovery and late sensory function recovery following surgical decompression.

One limitation of our study was the heterogeneity of our patient group. Although stringent criteria were used to recruit study participants, the symptoms of CSM in patients ranged in severity. It is unclear how the variability in symptom severity affects the absolute concentration of NAA in both the motor and sensory cortices. Another potential limitation of our study was the reproducibility in the placement of the MRS voxel in both the motor and sensory cortices. To minimize placement error in follow-up scans, we employed projections of the position of the voxel in 3 orientations from the baseline scan for each patient as a reference. However, differences in head orientation as well as subject motion between the short time that the T2-weighted localizer scans were acquired and the voxel placement and spectroscopy acquisition could have led to small errors in voxel placement.

\section{Conclusions}

To the best of our knowledge, this study is the first to characterize metabolic changes in the motor and sensory cortices following surgery in patients with CSM. These results may aid clinicians in counseling patients regarding the tempo and course of neurological recovery following surgery. Specifically for sensory complaints, our results suggest that at the 6-week follow-up, it may be too early to predict or prognosticate on the extent of recovery. For motor recovery, rehabilitation efforts should target motor function early in the postoperative course, as the greatest clinical and metabolic gains occur in the first 6 weeks following surgery. Ongoing and future studies should investigate metabolic changes and axonal integrity at various segments in neurological pathways beginning at the site of injury and ultimately comparing results found in both motor and sensory cortices.

\section{Acknowledgments}

We thank all participants involved in volunteering their time to be part of this study. We also thank the Canadian Institute of Health Research (CIHR) for funding.

\section{References}

1. Al-Tamimi YZ, Guilfoyle M, Seeley H, Laing RJ: Measurement of long-term outcome in patients with cervical spondylotic myelopathy treated surgically. Eur Spine J 22:25522557, 2013

2. Bartha R, Drost DJ, Williamson PC: Factors affecting the quantification of short echo in-vivo 1H MR spectra: prior knowledge, peak elimination, and filtering. NMR Biomed 12:205-216, 1999

3. Berger JR: Medical myelopathies, in Simeone FA, Rothman FH (eds): The Spine, ed 6. Philadelphia: Saunders, 2011, Vol 2

4. Bernhardt M, Hynes RA, Blume HW, White AA III: Cervical spondylotic myelopathy. J Bone Joint Surg Am 75:119-128, 1993

5. Chatham JC, Blackband SJ: Nuclear magnetic resonance spectroscopy and imaging in animal research. ILAR J 42:189-208, 2001

6. De Stefano N, Matthews PM, Arnold DL: Reversible decreases in N-acetylaspartate after acute brain injury. Magn Reson Med 34:721-727, 1995

7. Demougeot C, Garnier P, Mossiat C, Bertrand N, Giroud M, Beley A, et al: N-acetylaspartate, a marker of both cellular dysfunction and neuronal loss: its relevance to studies of acute brain injury. J Neurochem 77:408-415, 2001

8. Dong Y, Holly LT, Albistegui-Dubois R, Yan X, Marehbian J, Newton JM, et al: Compensatory cerebral adaptations before and evolving changes after surgical decompression in cervical spondylotic myelopathy. J Neurosurg Spine 9:538-551, 2008

9. Ellingson BM, Salamon N, Hardy AJ, Holly LT: Prediction of neurological impairment in cervical spondylotic myelopathy using a combination of diffusion MRI and proton MR spectroscopy. PLoS One 10:e0139451, 2015

10. Emery SE: Cervical spondylotic myelopathy: diagnosis and treatment. J Am Acad Orthop Surg 9:376-388, 2001

11. Good DC, Couch JR, Wacaser L: "Numb, clumsy hands" and high cervical spondylosis. Surg Neurol 22:285-291, 1984

12. Holly LT, Dong Y, Albistegui-DuBois R, Marehbian J, Dobkin B: Cortical reorganization in patients with cervical spondylotic myelopathy. J Neurosurg Spine 6:544-551, 2007

13. Kassem MN, Bartha R: Quantitative proton short-echo-time LASER spectroscopy of normal human white matter and hippocampus at 4 Tesla incorporating macromolecule subtraction. Magn Reson Med 49:918-927, 2003

14. Kowalczyk I, Duggal N, Bartha R: Proton magnetic resonance spectroscopy of the motor cortex in cervical myelopathy. Brain 135:461-468, 2012

15. Molina V, Sánchez J, Sanz J, Reig S, Benito C, Leal I, et al: Dorsolateral prefrontal N-acetyl-aspartate concentration in male patients with chronic schizophrenia and with chronic bipolar disorder. Eur Psychiatry 22:505-512, 2007

16. Moussellard HP, Meyer A, Biot D, Khiami F, Sariali E: Early neurological recovery course after surgical treatment of cervical spondylotic myelopathy: a prospective study with 2 -year follow-up using three different functional assessment tests. Eur Spine J 23:1508-1514, 2014

17. Salamon N, Ellingson BM, Nagarajan R, Gebara N, Thomas A, Holly LT: Proton magnetic resonance spectroscopy of human cervical spondylosis at 3T. Spinal Cord 51:558-563, 2013

18. Tracy JA, Bartleson JD: Cervical spondylotic myelopathy. Neurologist 16:176-187, 2010

19. van der Graaf M: In vivo magnetic resonance spectroscopy: basic methodology and clinical applications. Eur Biophys J 39:527-540, 2010

20. Voskuhl RR, Hinton RC: Sensory impairment in the hands secondary to spondylotic compression of the cervical spinal cord. Arch Neurol 47:309-311, 1990

21. Wang MC, Kreuter W, Wolfla CE, Maiman DJ, Deyo RA: 
Trends and variations in cervical spine surgery in the United States: Medicare beneficiaries, 1992 to 2005. Spine (Phila

Pa 1976) 34:955-963, 2009

22. Yousry TA, Schmid UD, Alkadhi H, Schmidt D, Peraud A, Buettner A, et al: Localization of the motor hand area to a knob on the precentral gyrus. A new landmark. Brain 120:141-157, 1997

\section{Disclosures}

The authors report no conflicts of interest concerning the materials or methods used in this study or the findings specified in this paper.

\section{Author Contributions}

Conception and design: Duggal, Goncalves, Bartha. Acquisition of data: Goncalves, Doyle-Pettypiece. Analysis and interpretation of data: Duggal, Goncalves, Stevens, Bartha. Drafting the article:
Goncalves. Critically revising the article: Duggal, Goncalves, Stevens, Bartha. Reviewed submitted version of manuscript: all authors. Approved the final version of the manuscript on behalf of all authors: Duggal. Statistical analysis: Goncalves. Administrative/technical/material support: Goncalves, Stevens, DoylePettypiece, Bartha. Study supervision: Duggal, Bartha.

\section{Supplemental Information}

\section{Previous Presentations}

Portions of this work were presented at the 2015 Annual Conference of the International Society for the Advancement of Spine Surgery held in San Diego, California, on April 15-17, 2015.

\section{Correspondence}

Neil Duggal, Division of Neurosurgery, University Hospital, LHSC, 339 Windermere Rd., London, ON N6A 5A5, Canada. email: neil.duggal@1hsc.on.ca. 